\title{
Advanced Integrated Future Vehicle Telematics System Concept Modeling
}

Ashmika Agarwal ${ }^{\star}$ and Vinay Yadav

Department of Mechanical, AISECT, Bhopal, Madhya Pradesh, India

*Corresponding author: Ashmika Agarwal, Department of Mechanical, AISECT, Bhopal, Madhya Pradesh, India, Tel: 0755 676 6100; E-mail: ashmikaagarwal@hotmail.co.uk

Received date: June 27, 2017; Accepted date: August 29, 2017; Published date: August 31, 2017

Copyright: $\odot 2017$ Agarwal A, et al. This is an open-access article distributed under the terms of the creative commons attribution license, which permits unrestricted use, distribution, and reproduction in any medium, provided the original author and source are credited.

\begin{abstract}
A paper presented on the futuristic model of telematics system which will not only provide the vehicle user guidance, emergency or road side assistance or vehicle diagnostic/ health report on data captured but will also provide in much more deeper level a real time risk of any failure or service requirement in a vehicle. This prediction will be made by highly sophisticated and very well integrated failure predictions of all the components in that vehicle of the particular lot subjected the real loads due to driving behavior the vehicle was subjected from $0 \mathrm{~km}$ till date. This research will also establish the level of traceability, fault detection, data integration and system integration at all tiers levels between the Vehicle manufacture to its end suppliers for achieving the best possible predictions. Later on will be developed as a platform for reliability based real-time designing of systems and components. This paper will also talk about the overall service system requirements in today's world and changes for adaptability in it.

Although an expensive affair currently, similar disintegrated systems are well adopted in Developed nations, but how and to what extent the developing nations such as Indian industry can better adopt and use this model/ technology at a faster pace. The roles of effective production systems, safety, service, ERP, EDI and Quality systems their integration will be discussed in much more details. Not only applications such as vehicle but use of this model in any kind of machine and its maintenance will be talked about. A need for real time data based designing approach which will be required in this revolutionary will be realized in this future telematics world.
\end{abstract}

Keywords Telematics; Automotive industry; Prediction; Reliability; Data analysis; System modeling

\section{Introduction}

Today's world of automotive telematics industry can be clearly bifurcated between the electronic vehicles and self-driven vehicle technologies. Telematics has shown is true potential for both the generations of vehicles and is growing to further penetrate deep within.

Currently, fuel monitoring, Eco driving, Vehicle tracking, Geo fencing, entertainment and broadcasting can be generally seen on passenger vehicle and fleets. Further, remote diagnostics, Insurance telematics are other upcoming areas being worked on. Building these systems for sustainable future will however depend on the services offered, extent of vehicle and system integration, reliability studies and modeling, low cost and ease to use devices.

This research has been performed on the existing telematics and vehicle systems, futuristic projected trends on the industry by key industry consultant such as Deloitte (Figure 1), study of mathematical modeling being worked on in other researches, industrial experience on duty cycle analysis and predictions, high cost and complex systems being use in sophisticated machines, current applications, etc.

An attempt has been made to come up with an integrated model which could be used across various sector in near future of automotive transportation industry providing not only user but all related sector an equal advantage further enhancing the road map with the divergent progressive model development [1-5].

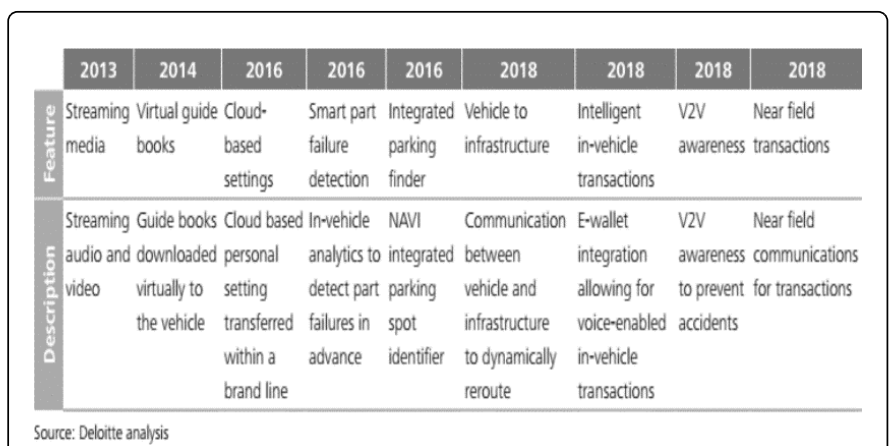

Figure 1: Forecasted features of telematics system. 


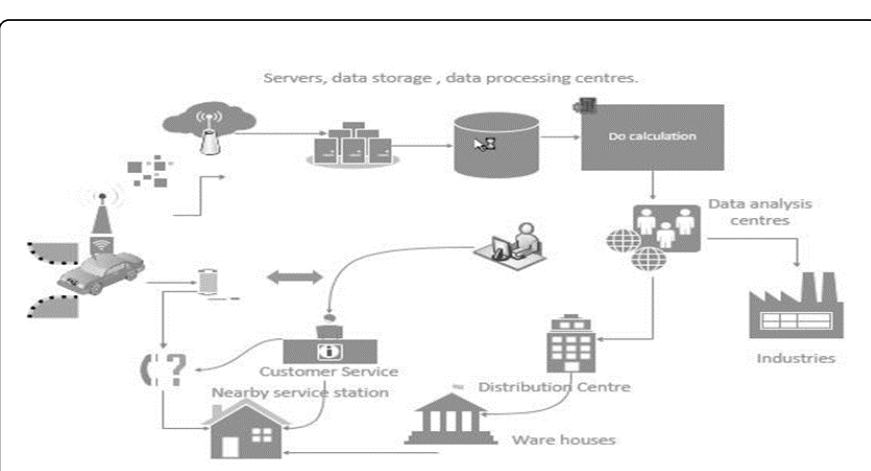

Figure 2: High level model.

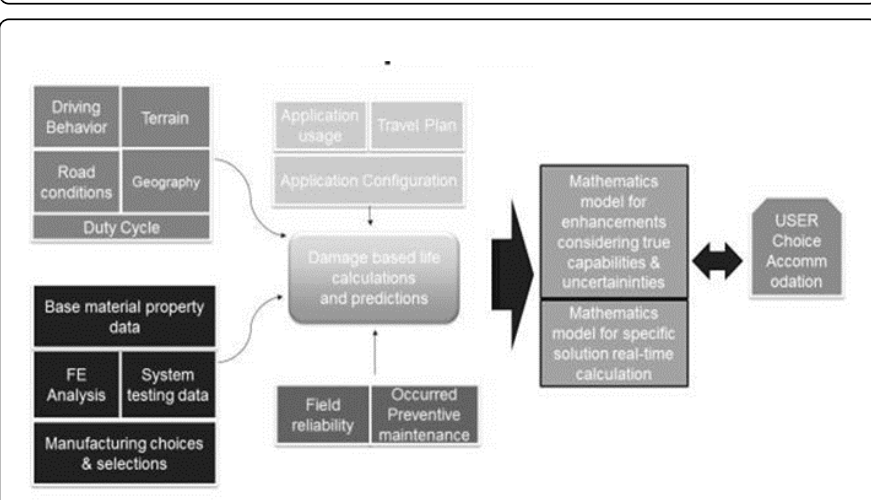

Figure 3: Concept Model for engineering reliability based predictions.

\section{Understanding Variables and Challenges}

- What we expect from the system: System is able to calculate damage on parts and recommend serviceability, usability and availability

- System is able to locate nearby service station and with it in emergency will limit the speeds of the vehicle.

- System able to check for the inventories and labor availability at next service station while giving the estimate of time required for services.

- System is able to calculate damage which will occur on the chosen route and can recommend for less damage routes, with min time and min distance route.

- System captures the available feature of telematics going in markets.

- System warns for rash driving behavior.

- System integrates with cloud and mobile for user interface.

- Feedback to the OE and its supplier on failure behavior and need for more robust design or manufacturing process.

- Communication channel to service stations and emergency stations.

- VOIP connectivity

- Data can further be refined and use for other service sectors which will benefit from it.

- What will be required to achieve expectation?
- All parts should have their engineering materials or damage cycle behavior attached; possibility can be seen to check for attaching these as metadata by the end suppliers itself.

- Integrated system required for vehicle and parts diagnostics.

- Capture the major contributors at first of failure then get with others.

- Firstly, if things can be calculated from given features or can be derived, it should be done. Secondly, use of sensors and loggers. And lastly, use theoretical estimator with practical variable in it like Weibull, Paris model etc. [6-8].

- Cases can be made on nature of vehicle for its service warning. like ambulance etc. which cannot suffer even one percent failure has will be quiet conservative when compared to passenger vehicle running city where an individual expect it to be more economic for taking chances of corrective measure rather than preventive i.e. repair after breakdown.

- Accuracy to improve with more data collection on that particular road and scenario.

- Integrated ERP as per the min quantity inventory stock and auto PO to OEM aftermarket sales. Visibility to AFM for consumption level of parts and future input to reliability with may be service $\mathrm{kms}$ details levels [8-10].

- Understanding the variables: Driver behavior, Environment behavior, Design limitations: product design, materials, manufacturing process, Mathematics of reliability and mapping to service, Vehicle ECU and telematics, Supply chain systems, Supply and demand analysis of after sales, Traceability of parts, And various Failure modes and their masking effects. Economic and infrastructure variables - Developing nations, still on top, Consumer behavior, Low cost oriented market, Commercial driver - very low wages and living, Road conditions, Diverse terrains, Less control on usability of a vehicle [10-15].

\section{Integrated high level model}

Electronic engine vehicle will be a must have system, where basic sensors are connect and can be read by the engine control units. Further this data taken on an appropriate interval need to be transferred to the computing units either within the system or on could servers. This data can then be cleaned and pre-processed by a preprocessor where auto programs will clean and process the data into useable form. Then this preprocessed data can be pulled by appropriate data centers for specific analysis. The preprocessor will also provide instantaneous alarms and critical information on the basis of auto programmer. Additional analysis, on demand basis will be sent from the data centers as requested.

These data centers will have link to Distribution centers, service centers for data exchange. This data can be related to inventory carrying levels at service center or field data for failures etc. Data centers will also be associated to various industries and service sectors seeking for this data or looking for a valuable analysis on this data. Data centers will be using high level of analysis software's, especially meant to deal with huge data with discretion individually and integrated analysis at other times. Later, these centers will also become a repository centers for a meaningful data with some trends which will later be helpful for prediction purpose and hence will even drive changes and improvement in systems.

With the information available to user for nearby service centers and facilities available, they will be able to select the centers and can 
directly communicate. Information which will be received by user from the service centers may include price, availability of parts suspected replacement, time taken for service, estimated wait time after their confirmation. Based on options most suitable, a value oriented parameter, preprocessor with GPS modules may suggested them time taken to reach the center and based on which the de-rate value suggested for their vehicles [15-18]. The calculation will also predict worst case scenarios where the engine will stop, if high risk observed and emergency service vehicle needs to be called on the spot.

The high level, 50,000 $\mathrm{ft}$ model which is derived can be seen in the Figure 2 and 3.

\section{Sub Systems, Concept and Math's}

\section{Sub system - user interface and vehicle level}

User need know in very simple and short language about the problem, remedies, risk level and option available to him. Ease of system should be that while driving even a driver can understand, choose and use these options without any significant road risk.

Vehicle inbuilt specific device are always an option. Although, low cost options will be Mobile connection to OBDII /CAN device $[19,20]$ Mobile devices can have apps programed to provide interface between user, vehicle and data center. Options such as VoIP will further enhance this option. Mobile interface to data analytic center and representative thru cloud based server or direct can depend on the app. Mobile interface to nearby service center thru cloud or data centers will further connect the personnel. User must have choice based on predictions and input options based on related prediction considerations. Rank percentage method, probability risk calculation ranking etc. can be suggest top 10 risk momentary risk he might be associated with it and it's mitigation suggested [21-25]. Once he has taken this choices, mitigation plan can be brought up for further actions and related choice within. In case of emergencies further protocol can be generated to call for an emergency to the appropriate agencies based on fault code obtained and instantaneous diagnostics models.

Mathematical model for choice in simple concurrent and concurrent-chains schedules and the cumulative decision model (CDM) proposed by Christensen and Grace (2010) and Grace and McLean $(2006,2015)$ which provides a good description of withinsession acquisition, and correctly predicts the effects of initial and terminal-link schedules in steady-state designs (Grace, 2002a), can be further consider while development.

Similarly, other sub systems which are defined are as follows -

- Sub System - Product design, Production Systems, Processing, Quality and Reliability

- Sub System - Computing and Analysis, Data and Information centers.

- Sub System - Service and Distribution channels

- Sub System - Communication

Like Weibull analysis of field data to predict life in usage and failure rates will be used. Correlation with Finite element analysis, test data's, material data, production methodology used related parameters, variability observed etc. will be variables attached to the parts or defining it in our model. These variable will flow through distribution centers and systems like EDI along with unique number identification the parts or assemblies [26-29]. Probability and Weibull equations will give the foundation of calculations. Although, other mathematical models are also available and should be establish for appropriate usage.

Computation and analysis may further use data analysis, engineering mathematical analysis tools such as nCode, or Mathworks (a product of Matlab). Later, even provides interface to use of 'Big Data' and machine learning modules which will further help. Optimized data, information flow should be established as per need and volume $[30,31]$ Service stations, distribution centers needs to have integrated softwares and should provide visibility on inventories and service analysis to the data centers, they should be linked with both user and data analysis centers. Communication channels will play a vital role in quality, time and information sharing.

\section{Applications}

Application: Warranty and Service, Marketing Network: Currently, fixed interval i.e. on basis of time or mileage defines service intervals for a vehicle. Additional Monitoring of driver's driving pattern, does he drive vigorous or smooth, slow or fast ; Environmental conditions such as dusty environment, ambient temperatures and pressures; Terrains and Altitude, uphill or downhill effects etc. play a vital role to which an application is subjected and what effect does it have on it [32-36]. For this Current real time reliability for components constituting the systems should be known or benchmarked with a similar nature component. Damage due to above factors when monitored additionally helps calculating specific service intervals on real time basis. No longer a person who drives smooth slow need to for early replacements than required. OEs will have additional advantages for design, product quality and performance, getting the real need of the market of the region and delivering product as per it.

Application: Product design: With this we in Indian automotive industry will move towards more optimized reliability based designing. In India, which is so diverse, more specific product can be sold, or altered in nearby service stations, close to customer's needs [37-39]. This will not only monitor data during warranty periods but also at later stages. A feedback loop for design will help to control much better durable and sustainable designs as per life requirement in future. Moreover, these will drive towards more controlled and environmental friendly products.

Application: Safety: Driver and passenger safety will be enhanced based on diagnostics and early predictions. Fault codes are currently being used in most of the electronic vehicles. Further it will be supported by a remote data analysis team or centers for intimation of high probability of failure and getting it corrected beforehand will result in avoidance of mishaps. This will also be followed by giving user option of derating so that he reaches nearby service station with a calculated risk. New features may further be added to support civil safety and emergency services systems.

Application: Road Laws and Insurance: Real-time data gathered in this process can even be helpful to government and civil agencies such as Regional Transportation Offices by enabling them to monitor rash driving, violation of traffic rules, emission limit etc. A specific module can also enable their decision making while mapping driving profiles for a license seeker. In case of accidents or emergencies, laws dept. can look at real time data of the accused for justice. Insurance companies can use this for more justified decisions on renewal of insurance, incurred amount etc. can be governed by the appropriate tools on driving real time pattern - analysis. 
Application: Service market and Distribution centers: This module with the prediction of failure will recommend high associated probable part which may require replacement or repair to the fleet owner. He will be able to further locate nearby service centers on his way where this part is readily available. Availability of part etc. will be linked with the system used by distribution centers and further to the OEs ERP systems through EDI etc. [40,41] Unique part identification will also be associated with its metadata on the back hand servers. Driver will be able to have a pre booking of the part and an estimate time for service for replacement. He will also have choice of other service stations nearby and estimated amount of service and time. In cases of emergencies derating features can be switched while depending on the distance to be covered and severity of issue. Hence, fleet owners can better monitor and plan even in case of emergencies and downtimes for on time deliveries.

\section{Indian Markets and Cost Effectiveness}

In terms of consumption and trend for use of vehicles in India markets has been lagging in technology when compare to that of developed nations. Indian economy is Cost driven. Although, in past few years we have seen that consumers have started to pay a bit extra on electronics, esp GPS etc. and hence urge of getting more through electronics and information systems is already started. And in future this trend is ought to accelerate with telematics implementation.

Low cost solutions and more basic problem solving modules will help to bring this wave at the earliest. Integrated solutions will require a huge workforce to get involved in this industry. Especially dealing with monitoring, data collection, refinement and analysis. Being in country like India this work force cost will be low than any other developed nations and could very well support the economy. Devices to be used can be as simple as OBDII device connected via mobile device rather data logger or more complex inbuilt devices [42]. Going forward much more compact devices which might be inbuilt moreover defining the vehicle itself will be helpful especially in road transportation, traffic control, insurance and govt services industries. More optimization and standardization of factors may further optimize those devices, hence lower cost. High volumes sales due to huge consumer market will lower the cost further. Tool developments and ease of use systems for user direct analysis by user themselves will have an added advantage.

\section{Advantages, Limitations and Future Scope}

Real time data gathering, monitoring and analysis will not only be helpful to consumers but also help various other services sectors, government agencies, and not the least industries themselves to provide much more optimized and better solutions.

Low cost solutions will help a major telematics industry growth in developing nations, helping employment and technological advancements too. To start with small devices such as OBDII adapter integrated to a mobile interface device with apps such as Torque and later some fault analysis tools such as ScanTool or Scan Mater- ELM will all that is needed by consumer. Later this backhand data set can be evaluated by data analysis teams or person himself with appropriate tools and teams.

On back hand, a high potential growth for related industries and service centers lies. This can be converted to useful data as per customer need and provided to various sectors such as insurance, engineering centers and government sectors. Technology, quality and environment norms could be effectively monitored. As the feedback loop will be generates this will also help engineering advancements for better optimized designs. It will also reduce waste and will be helpful to controls environmental norms to great extent.

Predictions will be till be based on probability and decision will still be taken by user themselves. Hence the outputs and optimization of mathematical models will be based on this. Solutions may some time lead to the divergence which may not be desired and hence monitoring and high skilled people will be required for analysis part. Vehicles which might not be using these systems will reduce level of confidence on data. During a unique product launch these systems will give more predictive signals rather preventive ones but as more data is gathered and enough time is spent in field preventive prediction will gain confidence. Traceability misses might create ambiguity. More complex systems will need to be established and monitored. Electronics vehicles and above generation will benefit. Minor errors may create misleading results which need to be monitored and ruled out as outliers. Work force initially required establishing these systems. Later emergence of AI, IOT advancement will lead to solve more tough problems in seconds. Although it will be important to leave the choice of decision to user, these systems are meant for guidance strictly. Nothing yet has replaced a human brain.

In future, much more integration, standardization and optimization of the sub system to that of main systems will be required to handle a lot of meaningful data. Much more technological advance devices compact in design, low cost products and services will be required. Establishing network both Integrated or discrete need to work online and offline. Development of physical systems, data systems, information systems, analysis systems and their interface will require to be put attention into. Data analysis -gathering data, creating logical networks, establishing correlation between various data. And the most important will be selection and development of mathematical models in a more advance forms, so as to process authentic information with least variables and lowest possible processing time. More work on mathematics of prediction to highest level of accuracies while appropriate control and feedback loops will be the most desired research areas to be looked upon.

\section{Results}

Based on the model above, we did an exercise on some data logging and analysis on available logged data with available resources, keeping low cost scenario in mind. Local service center and company Advance technologies manufacturing and designing electronic circuit boards were involved for trials and their expertise.

On the way from Bhopal to Chiklod the data was logged by ELM wireless OBDII device on Beat $1.2 \mathrm{~L}$ passenger car, this device was connected to mobile device which has a interfacing app called Torque II, this is a freeware available on app stores. The data logged was being stored on cloud through mobile data connection. All the channel including GPS, temperatures, Engine andVehicle speed and other related channels being monitored by vehicle ECU were logged. This data file could be retrieved on mail, i.e. from the cloud on request the logged files were sent to users email address. The data was filtered on excel for further used. This data was with timestamp in it. Hence, timeseries type of calculation could also be performed. This mobile software was even able to diagnose the fault codes. Though, the data obtained was at low frequency of $0.4 \mathrm{~Hz}$ and hence only few analysis 
could be performed. Although, for higher accuracy of results much more capable device and interfaces may be required to be developed.

We took some industrial logged data on real automotive application at $10 \mathrm{~Hz}$ which proved to be more accurate than ours. And hence used this data for further model validation purpose at service, data analysis and communication level. This was done developing some programs and using existing available methods and apps. Since, this was a single set of defined data general analysis method worked, later more sophisticated analysis tools including Math works -Big-Data software will be required to handle huge data with lots of variable. We also benchmarked few sub activities which have recently started developing in related areas or at a discrete level. This exercise validated the model, provided learnability opportunities and open a lot of areas in which future work will be required.

\section{Conclusion}

A base model for futuristic advance telematics provides a frame work to start with. For developing countries like India there are cost effective solutions which can be worked out integrating various aspects of user interface, product design, service sectors like insurance, government agencies, road transportation etc.

More optimization and development of mathematical models can be taken up with the advancement. With the probabilistic models account for uncertainty in prediction will still remain at human level. Networking, algorithms, real time analysis, accuracy, integration, information speed and cost effective devices and solutions will play an important role for ease and acceptable implementation of such systems. Standardization of parameters at base level and transparency in information across the network will provide sustainable solution.

Applications such as insurance systems, road safety and licensing systems, design feedback and warranty systems, manufacturing systems etc. And flow of these standardized common parameters across with the product capturing the environment variables too as being used with a back integration will provide a controlled, effective and efficient future of automotive industry.

Ultimately, not only Industries but also associated service sectors and government agencies need to work on this system with a low cost and integrated approach together laying the foundation and advancement of telematics in developing economies achieving most out of it.

\section{References}

1. Lenk FSM, Malek MA (2010) Survey of Online Failure Prediction Methods. ACM Journal Name 1-68.

2. Hoffmann GA (2006) Failure prediction in complex computer systems: A probabilistic approach. Shaker Verlag.

3. Csenki A (1990) Bayes predictive analysis of a fundamental software reliability model. IEEE Transactions on Reliability 39: 177-183.

4. Ochieng WY, Quddus MA, Noland RB (2004) Positioning algorithms for transport telematics applications. Journal of Geospatial Engineering 6: 10-30.

5. Salfner F (2010) Predicting failures with hidden markov models. Department of Computer Science Humboldt University Berlin.

6. Murray JF, Hughes GF, Kreutz-Delgado K (2005) Machine learning methods for predicting failures in hard drives: A multiple-instance application. Journal of Machine Learning Research 6 : 783-816.
7. Karapiperis D, Birnbaum B (2015) Usage-based insurance and vehicle telematics: Insurance market and regulatory implications. NAIC and the center of Insurance Policy and Research.

8. Deloitte Consulting LLP (2012) "Connected vehicles enter the mainstream Trends and strategic implications for the automotive industry".

9. MIL-HDBK-472 maintainability predictions.

10. MIL-STD-756B Reliability Modelling and predictions.

11. MIL-STD-785B Reliability programme for systems and equipment, development and production.

12. MIL-HDBK-8882C System safety programme requirements.

13. MIL-STD-1388-1A Logistic support analysis.

14. MIL-HDBK-5F 1990.

15. IEC 362 Guide for collection of reliability, availability, and maintainability data from field performance of electronics items (First edition)

16. ETSI ETR 039 Human Factors (HF); Human factors standards for telecommunication applications

17. SAE AIR 4276 Survey results: Computerization of reliability, maintainability and supportability (RMandS) in design

18. Ntasis E, Gletsos M, Mouravliansky NA, Zacharaki EI, Vasios CE, et al. (2005) Telematics enabled virtual simulation system for radiation treatment planning. See comment in PubMed Commons below Comput Biol Med 35: 765-781.

19. Maniatis A, Konstantina SN (2005) Telematics enabled virtual simulation system for radiation treatment planning. Computers in Biology and Medicine 35: 765-781.

20. Rao K, Rastogi S, Upadhya H, Sanmani A, Hakeem SA, Badari Narayan K (2007) Damage tolerance analysis of aero structural components. Tata Consultancy Services.

21. Brock D (1987) Elementary Engg. Fracture Mechanics. Martinus Nijhoff Pub.

22. Sih GC(1973) Hand book of SIF. Lehigh University Pennsylb USA

23. Tada H SIF and analysis of cracks Del research Hellertow.

24. Rook DP (1976) Cartwright DJ Compendium of SIF, HMSO, London.

25. Paris PC (1961) A rational analytic theory of fatigue. The trends in Engg. 13.

26. Fatigue Crack Growth Computer Program "NASGRO" VERSION 3.0. JSC-22267B, Reference-manual, and Oct.1999.

27. ReliaSoft Corporation (2007) Accelerated Life Testing Reference, Tucson, AZ: ReliaSoft Publishing.

28. Taylor S, Rupp C, Johnson D, Farrar C (2016), Avitabile P Failure Prediction In Composite Plates With Impact-Induced Damage. ImacXxii-Conf, Society for Experimental Mechanics, Inc.

29. Farrar CR (2003) Damage Prognosis: Current Status and Future Needs. Los Alamos National Laboratory report LA-14051-MS.

30. Robertson AN, Sohn H, Bement MT, Hunter NF, Liu C, et al. (2003) Damage Diagnosis and Prognosis for Composite Plates. Proceedings of the 21st International Modal Analysis Conference, Orlando, FL.

31. Intel White Paper In-Vehicle Telematics "Designing Next-Generation Telematics Solutions" 2015.

32. BusinessWire (2014) Global Commercial Telematics Subscribers will Reach Nearly 25 Million in 2014, According to ABI Research.

33. eMarketer, (2014)Smartphone Users Worldwide Will Total 1.75 Billion in 2014.

34. Usage-Based Insurance and Telematics (2015) National Association of Insurance Commissioners.

35. Visiongain report, "Connected Car Market 2015-2025".

36. Frank M, Moore G (2010) The Future of Work: A New Approach to Productivity and Competitive Advantage. Technology Solutions.

37. Caudoux M, Facchinetti ML, Raynal R (2014) Automotive stamped part fatigue design. Issue- MATEC Web of Conferences 12, FDMD II - JIP 2014 - Fatigue Design and Material Defects, Article Number 04021. 
Citation: Agarwal A, Yadav V (2017) Advanced Integrated Future Vehicle Telematics System Concept Modeling. Global J Technol Optim 8: 215. doi:10.4172/2229-8711.1000215

Page 6 of 6

38. Halfpenny A, Anderson R, Lin X (2015) Isothermal and ThermoMechanical Fatigue of Automotive Components. SAE Technical Paper.

39. Bennebacha $\mathrm{M}$, Cawte $\mathrm{R}$ Using a common Test and Simulation environment to optimize the Durability Process and verify the result. nCode international

40. Galtier A, Cugy P, Maronne E, Yoshida Y (2003) Integration of process operation in the fatigue calculation of sheets structural parts. SAE Technical Paper.
41. Driving for Work Using Telematics (2017). The Royal Society for the Prevention of Accidents (RoSPA, Scotland).

42. Using Telematics to Improve Driving for Work Safety: A Good Practice Guide( 2017). The Royal Society for the Prevention of Accidents (RoSPA, Scotland). 